\title{
Substrate-Mediated Intermolecular Hybridization in Binary Phthalocyanine Superstructures
}

\author{
Arrigo Calzolari, ${ }^{*},+\perp$ Wei Jin, ${ }^{\S}$ Janice E. Reutt-Robey, ${ }^{\S}$ and Marco Buongiorno Nardelli $*,,, \|$ \\ National Center on nanoStructures and bioSystems at Surfaces (S3) of CNR-INFM, 41100 Modena, Italy, \\ Department of Physics, North Carolina State University, Raleigh, North Carolina 27695, Department of \\ Chemistry and Biochemistry, University of Maryland, College Park, Maryland 20742, and CSMD, \\ Oak Ridge National Laboratory, Oak Ridge, Tennessee 37831
}

Received: August 24, 2009; Revised Manuscript Received: December 10, 2009

\begin{abstract}
Using a combination of calculations from first principles and scanning tunneling microscopy, we have investigated the interplay between substrate-mediated and intermolecular interactions in the formation of highdensity checkerboard binary superstructures from the codeposition of phenyl and perfluorophenyl Znphthalocyanines $\left(\mathrm{ZnPc}-\mathrm{F}_{16} \mathrm{ZnPc}\right)$ on the $\mathrm{Ag}(111)$ surface. The analysis of the electronic structure of the interface shows the essential role played by the substrate in the formation of the molecular layer and opens the way toward the development of tailored surfaces for advanced supramolecular design.
\end{abstract}

\section{Introduction}

Intermolecular interactions at surfaces drive rich structural diversity that promises exciting applications of molecular nanoscience. ${ }^{1}$ In particular, aromatic coordination complexes, such as metal phthalocyanines (MPc's), deposited on metal substrates are prototypical models of semiconductor/metal interfaces for molecular electronics applications., ${ }^{2,3}$

Despite the enormous interest that these ideas have spawned in recent years, it remains unclear whether structural diversity translates to functional diversity, especially when intermolecular interactions are noncovalent. Moreover, the effect of the molecule-substrate interaction is not completely understood and the potential role of the interface in tailoring the electronic properties of the assembly remains to be explored. It is thus important to investigate situations where intermolecular interactions and/or substrate-mediated interactions have dramatic consequences for the electronic structure of molecular assemblies and can be used for the tailoring of surface properties for advanced supramolecular design.

Metal phthalocyanines (MPc's) are promising candidate materials for organic thin film electronics due to their chemical stability, electronic and optical properties, and film quality. ${ }^{2}$ MPc's easily self-assemble on metal (e.g., $\mathrm{Cu}, \mathrm{Ag}, \mathrm{Au}$ ) surfaces, forming stable and ordered two-dimensional layers, ${ }^{4-6}$ with controlled electronic, ${ }^{7,8}$ magnetic, ${ }^{9}$ and optical ${ }^{10}$ properties. Phthalocyanines are typically hole-transport materials ${ }^{11}$ (i.e., donors), but they can be made into electron-transport materials by the simple chemical modification of replacing all peripheral hydrogen atoms with fluorine atoms. ${ }^{12}$ The availability of two molecules with virtually identical sizes and shapes, but with opposite electrical transport characteristics, is an intriguing one from the perspective of structure-property correlations in

* To whom correspondence should be addressed. Phone: +39-0403758737 (A.C.), +1-919-513-0514 (M.B.N.). Fax: +39-040-3758776 (A.C.), +1-919-513-4084 (M.B.N.). E-mail: arrigo.calzolari@democritos.it (A.C.), mbnardelli@ncsu.edu (M.B.N.).

National Center on nanoStructures and bioSystems at Surfaces (S3) of CNR-INFM.

* North Carolina State University.

$\S$ University of Maryland.

"Oak Ridge National Laboratory.

${ }^{\perp}$ Present address: Theory@Elettra Group, INFM-CNR DEMOCRITOS, c/o Sincrotrone Trieste-SS14, Km 163,5 Basovizza, I-34012 Trieste, Italy. molecular assemblies. A similar binary molecular system of $\mathrm{F}_{16} \mathrm{CoPc}$ and $\mathrm{Ni}$ tetraphenylporphine was found to display perfect intermixing, where the species alternated from site to site in an ordered 2D array. ${ }^{4}$

Indeed, specific halogen-hydrogen interactions may be exploited to drive the formation of novel, ordered, and dense molecular semiconductors, whose electronic properties are different from those of the single constituent Pc moieties. However, the early stages of the deposition and the formation of the first layer are crucial for the subsequent growth of the molecular film. Thus, the characterization of the moleculesubstrate and molecule-molecule interactions is a fundamental step to understanding the properties of hybrid semiconductor/ metal interfaces.

In this paper, we report on the study of the early stage of formation of a novel intermixed phthalocyanine-based layer $\left(\mathrm{ZnPc}-\mathrm{F}_{16} \mathrm{ZnPc}\right)$ on the $\mathrm{Ag}(111)$ surface using a combination of density functional theory (DFT) and scanning tunneling microscopy (STM). Our findings demonstrate a strong modification of the molecular electronic states due to the synergistic role of molecule-molecule and molecule-substrate interactions. In fact, both the density of states (DOS) and the conductive tunneling spectra reveal the formation of hybrid interface states. The resulting scenario allows us to identify an adsorption mechanism that is characterized by the transfer of charge between the adsorbate and the substrate, distinguishing the $\mathrm{ZnPc}-\mathrm{F}_{16} \mathrm{ZnPc} / \mathrm{Ag}(111)$ interface from a simple physisorption system.

\section{Technical Details}

Experiments. Experiments were carried out in an ultrahigh-vacuum (UHV) chamber (base pressure of $3 \times 10^{-9} \mathrm{~Pa}$ ) equipped with a dual organic molecular beam epitaxy source and a commercial STM (Omicrometer VT-STM). The Ag(111) substrate was prepared by several cycles of $\mathrm{Ar}^{+}$sputtering and annealing to approximately $500 \mathrm{~K} .{ }^{13} \mathrm{ZnPc}$ and $\mathrm{F}_{16} \mathrm{ZnPc}$ were coevaporated onto the $\mathrm{Ag}(111)$ surface from two separated $\mathrm{BN}$ crucibles at $660 \mathrm{~K}$ and $680 \mathrm{~K}$, respectively. The substrate was $\sim 5 \mathrm{~cm}$ from sources and kept at room temperature. The typical deposition rate was $0.25 \mathrm{~mL} / \mathrm{min}$. 
The STM images were acquired with a bias of $-0.839 \mathrm{~V}$ and a tunneling current of $40 \mathrm{pA}$. The bright/dim contrast, which corresponds to an apparent topographic difference of $0.02 \mathrm{~nm}$, is observed across a wide $(-2.0$ to $-0.4 \mathrm{~V})$ bias range. Scanning tunneling spectroscopy was performed in constant-current distance-voltage mode in order to minimize the likelihood of tip-induced molecular motion or chemical reaction. ${ }^{14-16}$

Theory. First-principles electronic structure calculations, based on density functional theory (DFT) approach, were performed with the Quantum ESPRESSO package, ${ }^{17}$ using plane waves and ultrasoft pseudopotential ${ }^{18}$ implementation. The plane-wave kinetic-energy cutoff for the wave functions (charge density) was 25 (200) Ry. The PBE-GGA ${ }^{19}$ exchange-correlation $(\mathrm{XC})$ functional was adopted. $\mathrm{A}(2 \times 2) k$-point grid was used for summations over the surface Brillouin zone (SBZ).

The unit supercell (Figure $2 \mathrm{a}$ ) contained one $\mathrm{F}_{16} \mathrm{ZnPc} / \mathrm{ZnPc}$ pair and four layers of $\operatorname{Ag}(111)$ with $(4 \sqrt{ } 3 \times 7)$ lateral periodicity, which corresponds to a $(20.13 \times 20.34) \AA^{2}$ cell, in agreement with the experimental findings. Slab replicas were separated by a vacuum region $\sim 14 \AA$ wide. Due to the absence of van der Waals interactions in DFT simulations, the vertical molecule-surface distance was not optimized; hence, the layer position was fixed at the height of $2.7 \AA$ above the substrate, as in similar MPc/Ag(111) systems. ${ }^{20,21}$

Maximally localized Wannier functions (MLWFs) ${ }^{22}$ were calculated by using the WanT code ${ }^{23}$ on the basis of the groundstate DFT electronic structure of the isolated binary $\mathrm{ZnPc} /$ $\mathrm{F}_{16} \mathrm{ZnPc}$ layer.

\section{Results}

Our STM measurements show that, when codeposited, ZnPc and $\mathrm{F}_{16} \mathrm{ZnPc}$ self-assemble into an ordered 2D superlattice with very few defects. This highly ordered structure extends over large domains, even crossing silver steps without interruption. Molecularly resolved images (Figure 1) reveal a checkerboard pattern, in which $\mathrm{ZnPc}$ and $\mathrm{F}_{16} \mathrm{ZnPc}$ are alternatively arranged along the substrate $[1 \overline{1} 0]$ and [11 $]$ ] directions. On comparison of the energy positions of the molecular orbitals in the binary and the single-species films, ${ }^{24}$ the bright molecule is assigned as $\mathrm{F}_{16} \mathrm{ZnPc}$, as confirmed by STS measurements and DFT calculations described below. One supramolecular unit cell ( $a$ $\left.=2.03 \pm 0.02 \mathrm{~nm}, b=2.01 \pm 0.02 \mathrm{~nm}, \theta=89 \pm 1^{\circ}\right)$ is superimposed on the STM image in Figure 1a. The submolecular resolution image shows one of the long axes of each molecule (i.e., fused rings) is now aligned with the close-packed direction of the substrate. Consequently, both $\mathrm{ZnPc}$ and $\mathrm{F}_{16} \mathrm{ZnPc}$ occupy identical adsorption sites on silver.

With respect to single-species deposition, the mix of $\mathrm{H}$-halogen terminations reduces the lateral repulsion forming intermolecular $\mathrm{H}$ bonds, ${ }^{25}$ which stabilize the adlayer and allow for the formation of ordered and close-packed 2D films. In fact, pure hydrogenated MPc $(\mathrm{M}=\mathrm{Fe}, \mathrm{Co}, \mathrm{Zn})$ layers on transitionmetal surfaces typically arrange with an average density of $\sim 0.3$ $\mathrm{mol} / \mathrm{nm},{ }^{2,4,20}$ whereas the density of the fully halogenated MPc films is still lower. ${ }^{26}$ The packing is closer $\left(\sim 0.4 \mathrm{~mol} / \mathrm{nm}^{2}\right)$ if partial halogenation is considered. In the present case, the alternate arrangement of $\mathrm{ZnPc}$ and $\mathrm{F}_{16} \mathrm{ZnPc}$ molecules in the checkerboard configuration maximizes the Coulomb attraction between the strongly electronegative fluorine atoms and the $\mathrm{H}$ of neighbor molecules, leading to a very dense $\left(\sim 0.5 \mathrm{~mol} / \mathrm{nm}^{2}\right)$ supramolecular film.

In the intermixed checker phase, the spectroscopy measurements (Figure 1b) are carried out successively on bright $\left(\mathrm{F}_{16} \mathrm{ZnPc}\right)$ and $\operatorname{dim}(\mathrm{ZnPc})$ molecules. A new electronic state
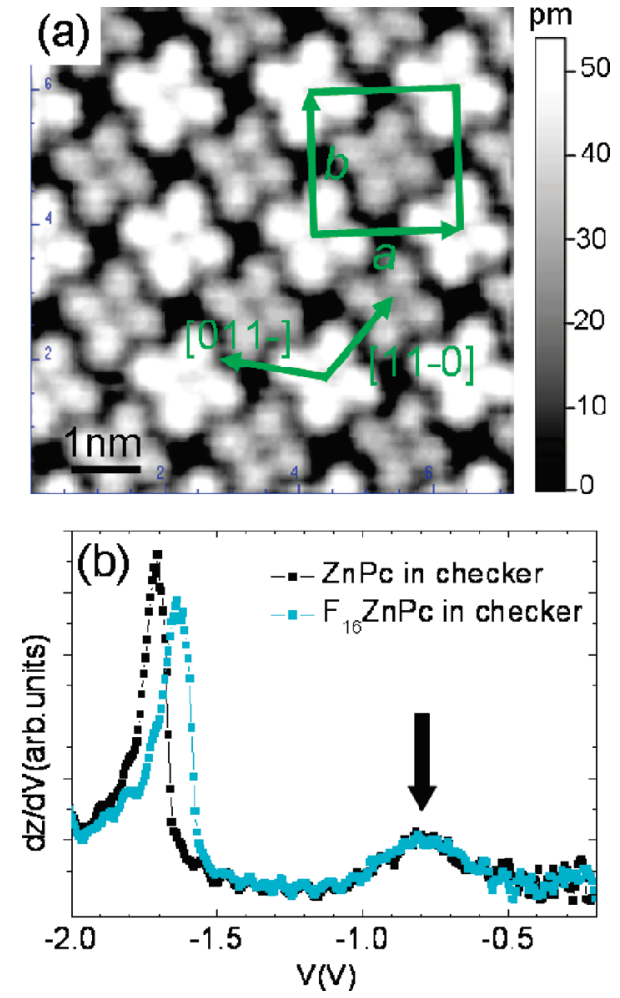

Figure 1. (a) STM image of one monolayer of the $\mathrm{ZnPc} / \mathrm{ZnPcF}_{16}$ intermixed phase with submolecular resolution. (b) Tunneling spectra measured on different species in the codeposited checker phase.

(vertical arrow), not distinguishable when single species are deposited, appears for both moieties at $-0.8 \mathrm{~V}$, while HOMO's are shifted to -1.75 and $-1.65 \mathrm{~V}$ for $\mathrm{ZnPc}$ and $\mathrm{F}_{16} \mathrm{ZnPc}$, respectively. The emergence of this feature is a clear fingerprint of electronic rehybridization at the interface.

To investigate the intimate nature of the electronic states close to the Fermi level, we undertook a massive theoretical study of the binary $\mathrm{F}_{16} \mathrm{ZnPc} / \mathrm{ZnPc}$ layer on $\mathrm{Ag}(111)$ by means of firstprinciples DFT simulations. The substrate-commensurate checkerboard arrangement of $\mathrm{F}_{16} \mathrm{ZnPc}$ and $\mathrm{ZnPc}$ is reproduced in the simulated STM image ${ }^{27}$ displayed in Figure 2b. Molecular contrast between $\mathrm{F}_{16} \mathrm{ZnPc}$ and $\mathrm{ZnPc}$ largely reflects the greater electron accumulation around fluorine. We notice also a $\mathrm{Zn}$ derived contribution (red arrows in Figure 2b) for both molecular species: this is the first proof that a molecule/substrate interaction took place. In fact, because of their square-like symmetry, the MPc molecular orbitals are easily classified according to the irreducible representation of the $D_{4 h}$ group. ${ }^{28}$ In the case of $\mathrm{Zn}$ phthalocyanine, the HOMO is singly degenerate and has an $\mathrm{a}_{1 \mathrm{u}}$ symmetry, which excludes direct metal-derived contributions. On the contrary, the LUMO is double degenerate and has a $2 \mathrm{e}_{\mathrm{g}}$ symmetry with a partial $3 \mathrm{~d}$ contribution by the inner $\mathrm{Zn}$ ion, ${ }^{29}$ similar to those presented in Figure 2b. Hence, the identification of a previously empty molecular state (LUMO) in the STM image for the occupied states of the interface is a marker of the electronic charge transfer from the surface toward the molecular layer.

The partial occupation of the former LUMO is confirmed also by analysis of the density of states (DOS) shown in Figure 2c. To focus on the molecular contributions, we projected the total DOS (shaded area) onto the two molecular subsystems, namely, $\mathrm{ZnPc}$ (black line) and the $\mathrm{F}_{16} \mathrm{ZnPc}$ (dashed cyan line). From the inset of Figure 2c that zooms on the energy region near the Fermi level, it is evident that the $2 \mathrm{e}_{\mathrm{g}}$ states of both Pc 

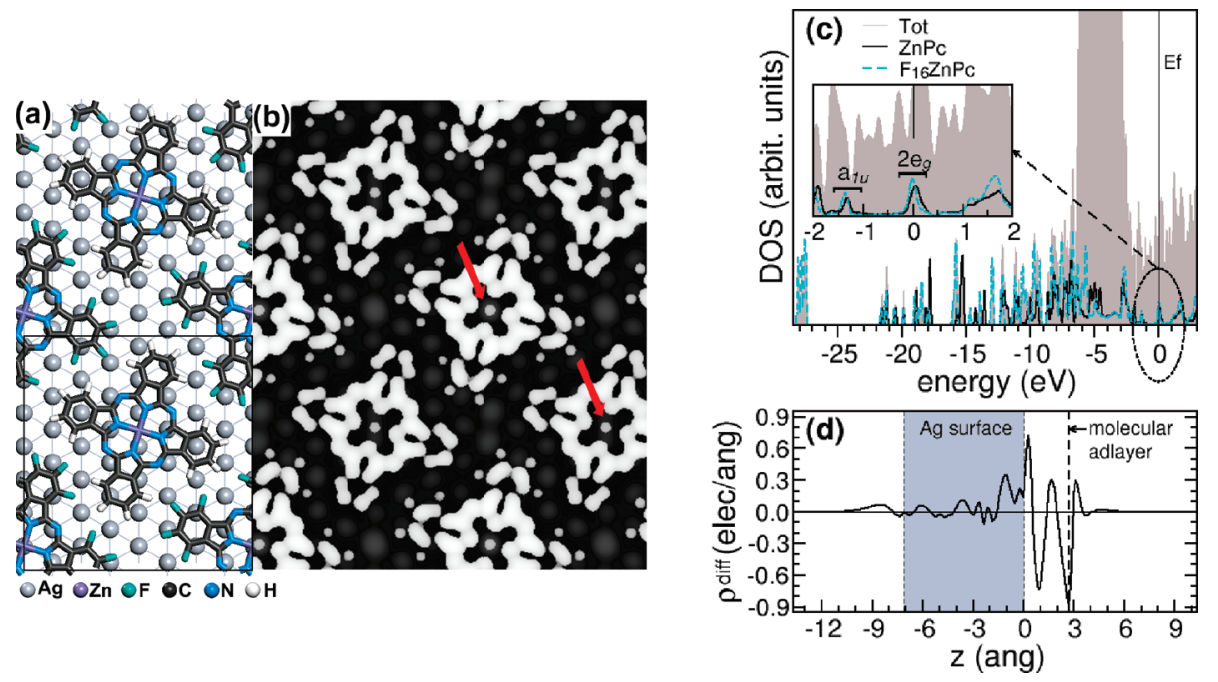

Figure 2. (a) Schematic representation of the $\mathrm{ZnPc} / \mathrm{F}_{16} \mathrm{ZnPc}$ supramolecular complex on the $\mathrm{Ag}(111)$ surface. The black box identifies the simulation cell. (b) Simulated constant current STM image for the hybrid interface collected at negative bias $(V=-500$ meV). Red arrows mark Zn-derived contributions. (c) Total (shaded area) and projected (straight and dashed lines) DOS for the molecule/surface interface. The inset zooms on the range near the Fermi level (vertical line). (d) Differential charge density profile ( $\left.\rho^{\text {diff }}=\rho^{\text {tot }}-\rho^{\text {surf }}-\rho^{\text {adlayer }}\right)$ along the [111] direction $(z)$, perpendicular to the surface.

moieties equally contribute to a new peak in the valence band of the final interface.

The hybrid states for this binary phthalocynine superstructure involve a diffuse metal-to-LUMO charge transfer that is quite distinct from chemisorption systems involving directional covalent bonds, such as the thiol/Au interface. In fact, except for a partial broadening of the molecular peaks, the two phthalocyanines maintain the main features of their isolated gasphase configurations. The delocalized $\pi$ character of the frontier orbitals interacts with the broad s band of silver, which acts as a surface free electron gas. This gives rise to a weak, but persistent, charge transfer from the metal to the $\mathrm{ZnPc}$ moieties that results in the occupation of the MPc's LUMO and the stabilization of the adlayer. The average charge transfer at the interface is shown in Figure 2d, which reports the differential charge density profile $\left(\rho^{\text {diff }}\right)$ along the [111] direction $(z)$, perpendicular to the surface, obtained as the planar average of the difference among the total charge density $\left(\rho^{\text {tot }}\right)$ and the isolated subsystems, that is, the bare surface $\left(\rho^{\text {surf }}\right)$ and the checker layer $\left(\rho^{\text {adlayer }}\right)$. The curve is noticeably different from zero only in the interstitial region, where a charge accumulation between surface and the molecule takes place. This weak-andextended coupling mechanism, previously proposed for aromatic metal-free molecules at surfaces such as PTCDA/Ag $(111)^{30}$ or pentacene/ $\mathrm{Cu}(100),{ }^{31}$ is now demonstrated also in the case of metal-coordinated systems, such as MPc's. In particular, for $\mathrm{ZnPc}$, the inner close-shell metal cation does not interact directly with the substrate, as proposed instead, for other phthalocyanines (e.g., CoPc, MnPc).

Notably, the presence of this molecular-derived peak at the Fermi level is in good qualitative agreement with the increase of the STS signal intensities observed in the experiments (Figure 1b). However, a direct quantitative comparison between theoretical and experimental results (e.g., the energy position between the calculated and the experimental peaks) should be considered with great care because of well-known limitations of the GGA exchange-correlation potential in the description of the electronic structure of such complex systems. The absence of quasi-particle corrections may affect the intrinsic gap of the molecules ${ }^{32,33}$ as well as their molecular orbital alignment with respect to the Fermi level of the metal surface.
Nonetheless, the most problematic incoherencies (e.g., wrong orbital ordering) have been observed for other Pc's (especially for $\mathrm{CuPc}^{34,35}$ ), for which the correlation effects are more relevant than in the $\mathrm{ZnPc}$ case. This, along with the presence of the metal surface that largely screens the correlation effects of the overall system and the similarities with other aromatic/metal interfaces, ${ }^{30,31}$ enforces the qualitative attribution of the LUMO-derived peak to the spectroscopic feature observed in the STS spectrum. Other hybrid XC functionals and/or more sophisticated techniques beyond the standard DFT (e.g., GW, CI) should be considered to solve these anomalies; however, their enormous computational requirements make these methodologies unfeasible to treat systems as large as the interface we are considering.

To decouple the intermolecular interaction from the substrate mediation, we characterized the $\mathrm{ZnPc}-\mathrm{F}_{16} \mathrm{ZnPc}$ binary phase in the absence of the silver substrate. The electronic properties of the layer can be best understood via the analysis of the individual bonding orbitals as represented by the maximally localized Wannier functions. To extract information on the bonding orbitals from a numerical procedure such as ours that relies on the periodicity of the simulation cell, one has to transform the Bloch orbitals, which are intrinsically delocalized, into localized functions. The core of our approach is to use maximally localized WFs for the system considered. These are the most natural choice for a set of localized orbitals that still span the same Hilbert space of the Hamiltonian eigenfunctions, and they allow to bridge plane-wave electronic structure and bonding orbital representation in a coherent fashion. In the case of an isolated system, the MLWFs become Boys localized orbitals $;^{36}$ therefore, our procedure is not tied to an extendedsystems formulation, but can equally well represent isolated molecules.

A few selected maximally localized Wannier functions for the checker configuration are reported in Figure 3. In particular, panels $\mathrm{a}-\mathrm{c}$ account for the electrostatic orbital polarization among neighbor molecules, that is, the formation of three $\mathrm{C}-\mathrm{H} \cdots \mathrm{F}$ bonds per (perfluorinated)phenyl group. Panel $\mathrm{d}$ shows an example of the intramolecular nonbonding interactions between two adjacent phenyl groups mediated by a central $\mathrm{N}$ atom. This confirms how complex the electrostatic distribution is, being the result of both inter- and intramolecular interactions. 

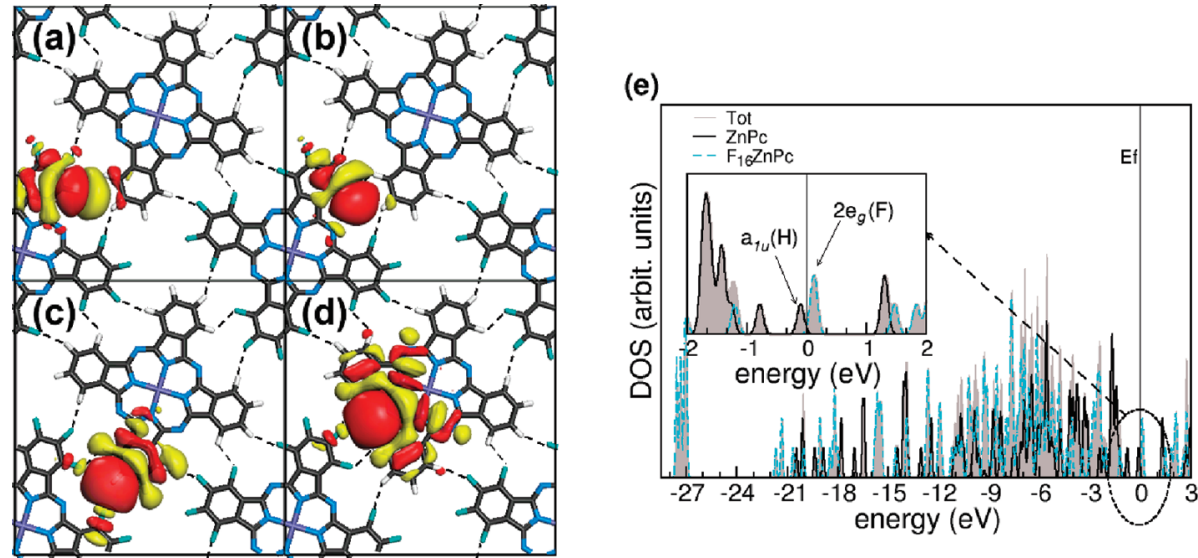

Figure 3. $(\mathrm{a}-\mathrm{d})$ Isosurface plots of selected MLWFs for the isolated (without substrate) $\mathrm{ZnPc} / \mathrm{F}_{16} \mathrm{ZnPc}$ assembly. Dashed lines identify the intermolecular H-bonding path. Atomic color labels follow Figure 2a. (e) Total (shaded area) and projected (straight and dashed lines) DOS for the molecule/surface interface. The inset zooms on the range near the Fermi level (vertical line).

Even though the intermolecular coupling is crucial for adlayer stabilization, this coupling does not lead to the formation of covalent bonds between Pc's. The DOS analysis reported in Figure $3 \mathrm{e}$ shows that the total spectrum is just the superposition of the two single molecule contributions. Due to the high electron affinity of the fluorine atoms decorating the macrocycle, the spectrum of $\mathrm{F}_{16} \mathrm{ZnPc}$ is rigidly shifted toward lower energy with respect to the $\mathrm{ZnPc}$ molecule. Thus, the HOMO of the checker is simply the $\mathrm{a}_{1 \mathrm{u}}$ state of $\mathrm{ZnPc}$ (labeled $\mathrm{H}$ in Figure 3e) and the LUMO is the $2 \mathrm{e}_{\mathrm{g}}$ of the $\mathrm{F}_{16} \mathrm{ZnPc}$ molecule (labeled $\mathrm{F}$ in Figure 3e). The degeneracy of the states is also maintained. At the interface, this shift is canceled by the screening effect of the metal surface. In the isolated layer, instead, the energy level of LUMO is shifted very close to HOMO, almost closing the gap, but no (partial) filling of the former empty orbital is observed.

We can conclude that the electrostatic intermolecular coupling does not directly generate the occupied state detected in the STS measurements (Figure 1b). Rather, the state reflects a molecule-substrate effect. This effect is, in fact, observed also in monomolecular films. However, in that case, repulsive interactions between molecules with the same terminations translate to a more distorted and mobile adlayer. ${ }^{22}$ Reduced registration with the substrate may attenuate the coupling peak in the STS spectra of pure films. In the binary complex, however, the added stabilization from the $\mathrm{C}-\mathrm{H} \cdots \mathrm{F}$ hydrogen bond network produces more resilient films for tunneling measurements, permitting the net identification of the sharp peak in the tunneling spectra.

On the other hand, because the conduction properties of molecular crystals are essentially the same as those of the component molecules $^{37}$ (i.e., the $\mathrm{ZnPc}-\mathrm{F}_{16} \mathrm{ZnPc}$ pair in this case), the DOS plot of Figure $3 \mathrm{e}$ is representative also of the bulk properties of the $\mathrm{ZnPc}-\mathrm{F}_{16} \mathrm{ZnPc}$ film. Thus, the strong closing of the bandgap in Figure $3 e$, though nondirectly comparable with the experimental one, qualitatively confirms that the perflorination of $\mathrm{ZnPc}$ is an effective doping strategy to obtain an n-type molecular semiconductor.

This interpretation is further confirmed by studying the electronic structure of the same checkerboard superstructure deposited onto a more inert substrate: the H-passivated Si(111) surface. The adsorption of atomic $\mathrm{H}$ saturates the dangling bonds of $\mathrm{Si}(111)$, making the surface an almost inert substrate. We considered a simulation cell with $\mathrm{H} / \mathrm{Si}(111)-(5 \times 3 \sqrt{ } 3)$ lateral
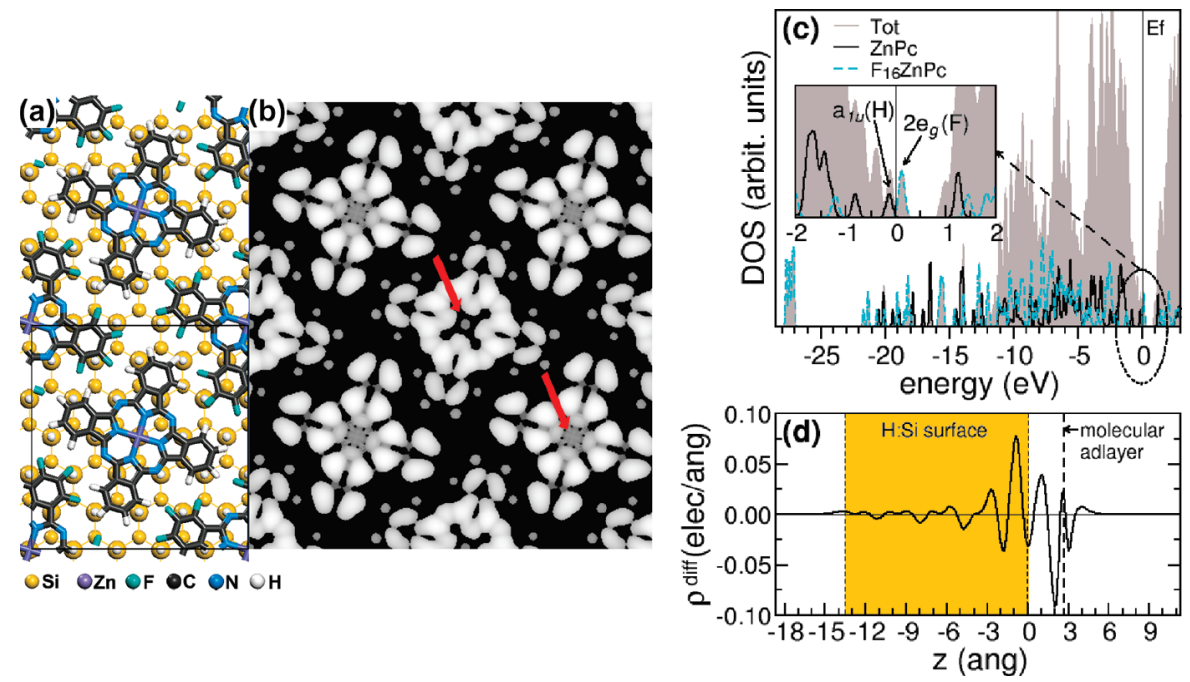

Figure 4. (a) Schematic representation of the $\mathrm{ZnPc} / \mathrm{F}_{16} \mathrm{ZnPc}$ supramolecular adlayer on the passivated H/Si(111) surface. The black box identifies the simulation cell. (b) Simulated STM image for the hybrid interface collected at negative bias $(V=-500$ meV) and constant current. Red arrows mark Zn-derived contributions. (c) Total (shaded area) and projected (straight and dashed lines) DOS for the molecule/surface interface. The inset zooms on the range near the Fermi level (vertical line). (d) Differential charge density profile $\left(\rho^{\text {diff }}\right)$ along the [111] direction $(z)$, perpendicular to the surface. 
periodicity, which corresponds to an almost squared $(19.19 \times$ 19.95) $\AA^{2}$ cell, and may host the molecular assembly with a very similar density (Figure $4 \mathrm{a}$ ).

The resulting interface has a completely different electronic structure with respect to the silver case. The main contribution to the simulated STM image (Figure 4b) stems from the HOMO state of the standing Pc's, while the LUMO states do not appear in the STM image: the minor Zn-ion contributions (arrows in Figure $4 b$ ) derive from low-lying energy states (e.g., $b_{1 g}$ ). The DOS (Figure 4c) does not present the (partial) occupation of the former LUMO states of phthalocyanines, observed in the presence of silver substrate. The projected DOS components (see inset) reproduce, instead, the shifted molecular DOS typical of the isolated layer (Figure 3e). Finally, the differential charge profile is 1 order of magnitude smaller than in the Ag case, a signature of weak polarization and dispersion effects at the interface, rather than a truly charge transfer process. This confirms the active role of the silver substrate in the adsorption of the resulting supramolecular layer.

\section{Conclusions}

In this study, we present a combined theoretical and experimental study of the structural and electronic properties of the hybrid organic/inorganic interface, resulting from the adsorption of phenyl and perfluorophenyl Zn-phthalocyanines on the $\operatorname{Ag}(111)$ surface.

We observed that codeposition of $\mathrm{ZnPc}$ and $\mathrm{F}_{16} \mathrm{ZnPc}$ gives an intermixed "checkerboard" phase. Hybridization of delocalized conjugated molecular orbitals with the quasi-free electron of the metallic surface generates a new occupied electronic structure for the intermolecular complex. Prominent in this new orbital landscape is a low-energy state, delocalized over the entire interface, which would likely contribute strongly to electrical transport processes in the intermixed films. This is a genuine interface effect that characterizes the formation and the properties of the first molecular layer. On the other hand, we expect that the strong intermolecular interactions will dictate the n-type semiconducting behavior of the thick molecular film.

Acknowledgment. The authors wish to acknowledge Daniel B. Dougherty for having brought this problem to their attention and for the scientific discussions that have ensued. William G. Cullen and Steven W. Robey are also acknowledged for technical and scientific discussions. This work has been supported, in part, by the BES, U.S. DOE, at ORNL (DE-FG0298ER14847 and DE-AC05-00OR22725 with UT-Battelle, LLC), the NSF-CCI Center for Molecular Spintronics (CHE-0943975), and the UMD MRSEC (NSF Grant DMR-05-20471). Calculations have been carried out at CINECA supercomputing facilities through a grant from CNR-INFM and NCCS-ORNL.

\section{References and Notes}

(1) Bart, J. V.; Costantini, G.; Kern, K. Nature 2005, 437, 671-679.

(2) Witte, G.; Wöll, C. J. Mater. Res. 2004, 19, 1889-1916.

(3) Nazin, G. V.; Qiu, X. H.; Ho, W. Science 2003, 302, 77-81.
(4) Hipps, K. W.; Scudiero, L.; Barlow, D. E.; Cooke, M. P. J. Am. Chem. Soc. 2002, 124, 2126-2127.

(5) Wakayama, Y. J. Phys. Chem. C 2007, 111, 2675-2678.

(6) Oison, V.; Koudia, M.; Abel, M.; Porte, L. Phys. Rev. B 2007, 75, 035428 .

(7) Song, F.; Wells, J. W.; Handrup, K.; Li, Z. S.; Bao, S. N.; Schulte, K.; Ahola-Tuomi, M.; Mayor, L. C.; Swarbrick, J. C.; Perkins, E. W.; Gammelgaard, L.; Hofmann, Ph. Nat. Nanotechnol. 2009, 4, 373-376.

(8) Calabrese, A.; Floreano, L.; Verdini, A.; Mariani, C.; Betti, M. G. Phys. Rev. B 2009, 79, 115446.

(9) Vitali, L.; Fabris, S.; Mosca Conte, A.; Brink, S.; Ruben, M.; Baroni, S.; Kern, K. Nano Lett. 2008, 8, 3364-3368.

(10) Kalyuzhny, G.; Vaskevich, A.; Ashkenasy, G.; Shanzer, A.; Rubinstein, I. J. Phys. Chem. B 2000, 104, 8238-8244.

(11) Bao, Z.; Lovinger, A. J.; Dodabalapur, A. Appl. Phys. Lett. 1996, 69, 3066-3068.

(12) Schon, J.; Bao, Z. J. Appl. Phys. 2001, 89, 3526-3528.

(13) Koudia, M.; Abel, M.; Maurel, C.; Bliek, A.; Catalin, D.; Mossoyan,

M.; Mossoyan, J.-C.; Porte, L. J. Phys. Chem. B 2006, 110, 10058-10062.

(14) Manandhar, K.; Ellis, T.; Park, K. T.; Cai, T.; Song, Z.; Hrbek, J. Surf. Sci. 2007, 601, 3623-3631.

(15) Kemerink, M.; Alvarado, S. F.; Muller, P.; Koenraad, P. M.; Salemink, H. W. M.; Wolter, J. H.; Janssen, R. A. J. Phys. Rev. B 2004, 70,045202 .

(16) Dougherty, D. B.; Maksymovych, P.; Lee, J.; Yates, J. T., Jr. Phys. Rev. Lett. 2006, 97, 236806.

(17) Quantum-ESPRESSO is a community project for high-quality quantum-simulation software, based on density functional theory and coordinated by Paolo Giannozzi. See: www.quantum-espresso.org.

(18) Vanderbilt, D. Phys. Rev. B 1990, 41, R7892-R7895.

(19) Perdew, J. P.; Burke, K.; Ernzerhof, M. Phys. Rev. Lett. 1996, 77, $3865-3868$.

(20) Alvarado, S. F.; Seidler, P. F.; Lidzey, D. G.; Bradley, D. D. C. Phys. Rev. Lett. 1998, 81, 1082-1085.

(21) Gerlach, A.; Schreiber, F.; Sellner, S.; Dosch, H.; Vartanyants, I. A.; Cowie, B. C. C.; Lee, T.-L.; Zegenhagen, J. Phys. Rev. B 2005, 71, 205425.

(22) Marzari, N.; Vanderbilt, D. Phys. Rev. B 1997, 56, 12847-12865.

(23) Ferretti, A.; Bonferroni, B.; Calzolari, A.; Buongiorno Nardelli, M. WanT code; available at www.wannier-transport.org. See also: Calzolari, A.; Marzari, N.; Souza, I.; Buongiorno Nardelli, M. Phys. Rev. B 2004, 69, 035108 .

(24) Peisert, H.; Knupfer, M.; Schwieger, T.; Fuentes, G. G.; Olligs, D.; Fink, J. J. Appl. Phys. 2003, 93, 9683-9692.

(25) Thalladi, V. R.; Weiss, H.-C.; Bläser, D.; Boese, R.; Nangia, A.; Desiraju, G. R. J. Am. Chem. Soc. 1998, 120, 8702-8710.

(26) Baski, A. A.; Fuchs, H. Surf. Sci. 1994, 313, 275-288.

(27) Tersoff, J.; Hamann, D. R. Phys. Rev. Lett. 1983, 50, 1998-2001.

(28) Liao, M. S.; Scheiner, S. J. Chem. Phys. 2001, 114, 9780-9791.

(29) The amount of $\mathrm{Zn}_{3 \mathrm{~d}}$ contribution to LUMO largely depends on the choice of the adopted XC functional. Different results are reported, for instance, in refs 28 and 32 or Ueno, L. T.; Machado, A. E. H.; Machado, F. B. C. J. Mol. Struct. (THEOCHEM) 2009, 899, 7178. Here, the symmetry analysis of molecular orbitals confirms the identification of the ZnPc LUMO state in the STM (and DOS) images.

(30) Eremtchenko, M.; Schaefer, J. A.; Tautz, F. S. Nature 2003, 425, 602-605.

(31) Ferretti, A.; Baldacchini, C.; Calzolari, A.; Di Felice, R.; Ruini, A.; Molinari, E.; Betti, M. G. Phys. Rev. Lett. 2007, 99, 046802.

(32) Nemykin, V. N.; Hadt, R. G.; Belosludov, R. V.; Mizuseki, H.; Kawazoe, Y. J. Phys. Chem. A 2007, 111, 12901-12913.

(33) Palammo, M.; Hogan, C.; Sottile, F.; Bagalà, P.; Rubio, A. J. Chem. Phys. 2009, 131, 084102.

(34) Calzolari, A.; Ferretti, A.; Buongiorno Nardelli, M. Nanotechnology 2007, 18, 424013.

(35) Marom, N.; Hod, O.; Scuseria, G. E.; Kronik, L. J. Chem. Phys. 2008, $128,164107$.

(36) Boys, S. F. Rev. Mod. Phys. 1960, 32, 300.

(37) Liozzi, L.; Santucci, S.; La Rosa, S.; Delley, B.; Picozzi, S. J. Chem. Phys. 2004, 121, 1883-1889.

JP908166J 